Determining Cross Sections for Reactions on Unstable Nuclei: A Consideration of Indirect Approaches

J. Escher, F. S. Dietrich

May 27, 2005

2nd Argonne/MSU/JINA/INT RIA Workshop - Reaction Mechanisms for Rare Isotope Beams East Lansing, MI, United States March 9, 2005 through March 12, 2005 
This document was prepared as an account of work sponsored by an agency of the United States Government. Neither the United States Government nor the University of California nor any of their employees, makes any warranty, express or implied, or assumes any legal liability or responsibility for the accuracy, completeness, or usefulness of any information, apparatus, product, or process disclosed, or represents that its use would not infringe privately owned rights. Reference herein to any specific commercial product, process, or service by trade name, trademark, manufacturer, or otherwise, does not necessarily constitute or imply its endorsement, recommendation, or favoring by the United States Government or the University of California. The views and opinions of authors expressed herein do not necessarily state or reflect those of the United States Government or the University of California, and shall not be used for advertising or product endorsement purposes. 


\title{
Determining Cross Sections for Reactions on Unstable Nuclei: A Consideration of Indirect Approaches
}

\author{
J. Escher ${ }^{1}$ and F.S. Dietrich \\ Lawrence Livermore National Laboratory, P.O. Box 808, L-414, Livermore, CA 94551, USA
}

\begin{abstract}
An indirect method for determining cross sections for reactions proceeding through a compound nucleus is presented. The appropriate theoretical framework for applications of this method is reviewed and theoretical and experimental challenges that need to be addressed in applications of the method are outlined. Two approximations are considered and their advantages and limitations are discussed.
\end{abstract}

Keywords: statistical compound-nucleus reactions, indirect methods, unstable nuclei PACS: 24.10.-i, 24.60.Dr, 25.40.Lw, 25.85.Ec

\section{INTRODUCTION}

A large number of nuclear reactions cannot be easily determined in the laboratory. Direct measurements encounter a variety of difficulties: The low-energy regime that is especially relevant for astrophysical reactions is often inaccessible and cross sections for charged-particle reactions become vanishingly small as the relative energy of the colliding nuclei decreases. Furthermore, many reactions involve unstable nuclear species which are too difficult to produce with currently available experimental techniques or too short-lived to serve as targets in present-day set-ups.

In order to overcome the experimental limitations, various indirect methods have been proposed in recent years. Coulomb Dissociation [1], e.g., has been used to extract cross sections for radiative-capture reactions, $A(a, \gamma) B$. In this approach, the Coulomb field of a highly charged target provides a virtual photon, which is absorbed by the projectile $B$. The cross section of the breakup $X(B, A a) X$ is much larger than the capture cross section and can be related to the latter via the principle of detailed balance. The ANC (Asymptotic Normalization Coefficient) Method [2] has been explored for low-energy radiative-capture reactions $A(a, \gamma) B$ which are dominated by processes occurring far outside the nuclear radius. The cross section of such a reaction depends on the asymptotic behaviour of the overlap function $I_{A a}^{B}$ for $B \rightarrow A+a$. The radial shape of $I_{A a}^{B}$ is well known, and its normalization, the ANC, can be determined via a peripheral transfer reaction that involves the same asymptotic overlap, e.g. $d+A \rightarrow b+B$, where $d=a+b$ and $B=A+a$. The Trojan-Horse method [3] provides a mechanism for circumventing the Coulomb barrier which is responsible for the very small cross sections in low-energy two-body reactions with charged projectiles, $A(a, b) B$. It does so by selecting a reaction $d+A \rightarrow b+B+c$ with $d=a+c$ and kinematic conditions such that $c$ can be considered a spectator in the reaction between $a$ and $A$ ('quasi-free scattering'). An approximate expression for the cross section of the three-body reaction then provides a link to the two-body reaction of interest and allows one to extract the energydependence of the latter. Thus it becomes possible to extrapolate absolute measurements carried out at higher energies to the relevant low-energy regime.

The focus of this contribution is an indirect method that complements the above approaches, the Surrogate Nuclear Reactions method. The Surrogate method combines experiment with reaction theory to obtain cross sections for compound-nucleus reactions involving difficult-to-produce targets. In this article, we discuss the Surrogate approach and two approximations to the method. In the next section the Surrogate idea is explained and the appropriate theoretical framework for describing a Surrogate reaction is outlined. In Section 3, we discuss briefly the WeisskopfEwing limit of the full Hauser-Feshbach theory and consider its implications for the Surrogate approach. In Section 4, we outline a new idea [4], which aims at extracting ratios of reaction cross sections from Surrogate experiments. An independent measurement of one of the reactions can then be used to obtain an unknown cross section. The "Ratio method" was recently employed to estimate the ${ }^{237} \mathrm{U}(\mathrm{n}, \mathrm{f})$ cross section [5]. We discuss some of the advantages and

\footnotetext{
${ }^{1}$ Email: escher1@1ln1.gov
} 


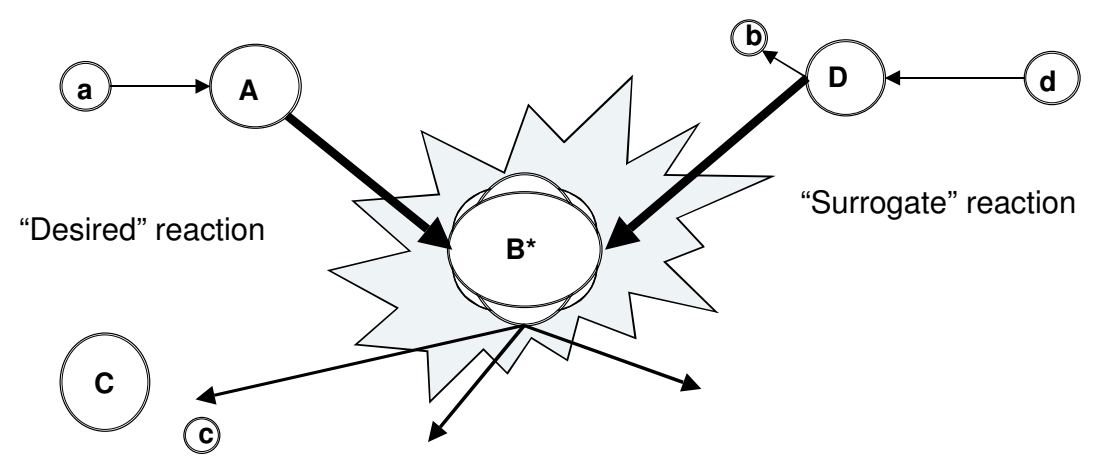

FIGURE 1. Schematic representation of the Surrogate reaction mechanism. The basic idea of the Surrogate approach is to replace the first step of the desired reaction by an alternative ("Surrogate") reaction that populates the same compound nucleus. The subsequent decay of the compound nucleus into the relevant channel can then be measured and used to extract the desired cross section.

limitations of this approach. Concluding remarks are given in Section 5.

\section{THE SURROGATE METHOD}

The Surrogate nuclear reaction technique is an indirect method for determining the cross section for a particular type of "desired" reaction, namely a two-step reaction, $a+A \rightarrow B^{*} \rightarrow c+C$, that proceeds through a compound nuclear state $B^{*}$, a highly excited state in statistical equilibrium (see Figure 1 ). In the Surrogate method, the compound nucleus $B^{*}$ is produced by means of an alternative ("Surrogate") reaction, here $d+D \rightarrow b+B^{*}$, and the reaction cross section is obtained by combining the calculated cross section for the formation of $B^{*}$ (from $a$ and $A$ ) with the measured decay probabilities for this state. The Surrogate technique is particularly valuable when the target of interest, $A$, is short-lived and a suitable Surrogate reaction involving a stable target $D$ can be identified. A simple version of the Surrogate idea was already used in the 1970s to estimate neutron-induced fission cross sections from transfer reactions [6]. More recently, this approach was refined [11] and applications to other reactions are now being considered, such as $(n, \gamma)$ reactions on s-process branch points [7].

\subsection{Hauser-Feshbach Formalism for Compound-Nucleus Reactions}

The formalism appropriate for describing compound-nucleus reactions is the statistical Hauser-Feshbach theory (see, e.g., chapter 10 of Ref. [8]). The average cross section per unit energy is given by:

$$
\frac{d \sigma_{\alpha \chi}^{H F}\left(E_{a}\right)}{d E_{\chi}}=\pi \tau_{\alpha}^{2} \sum_{J \Pi} \omega_{\alpha}^{J} \sum_{l s l^{\prime} s^{\prime} I^{\prime}} \frac{T_{\alpha l s}^{J} T_{\chi l^{\prime} s^{\prime}}^{J} \rho_{I^{\prime}}\left(U^{\prime}\right)}{\sum_{\chi^{\prime \prime} l^{\prime \prime} s^{\prime \prime}}^{\prime} T_{\chi^{\prime \prime} l^{\prime \prime} s^{\prime \prime}}^{J}+\sum_{\chi^{\prime \prime} l^{\prime \prime} s^{\prime \prime} I^{\prime \prime}}^{\int} T_{\chi^{\prime \prime} l^{\prime \prime} s^{\prime \prime}}^{J}\left(E_{\chi^{\prime \prime}}\right) \rho_{I^{\prime \prime}}\left(U^{\prime \prime}\right) d E_{\chi^{\prime \prime}}} .
$$

where it is assumed that the reaction proceeds to an energy region in the final nucleus described by a level density. Here $\alpha$ denotes the entrance channel $a+A$ and $\chi$ represents the relevant exit channel $c+C, E_{a}$ is the kinetic energy of the projectile, and $\lambda_{\alpha}$ is the reduced wavelength in the incident channel (the inverse of the wave number). The spin of the incident particle is $i$, the target spin is $I$, the channel spin is $\vec{s}=\vec{i}+\vec{I}$, and the compound-nucleus angular momentum and parity are $J \pi$. The statistical-weight factor $\omega_{\alpha}^{J}$ is $(2 J+1) /[(2 i+1)(2 I+1)]$. Similarly, the spin of the outgoing particle is $i^{\prime}$, the spin of the residual nucleus is $I^{\prime}$, and the channel spin for $\chi^{\prime}$ is $\vec{s}^{\prime}=\vec{i}^{\prime}+\vec{I}^{\prime}$. The transmission coefficients are written as $T_{\alpha l s}^{J}$ and $\rho_{I^{\prime}}\left(U^{\prime}\right)$ denotes the density of levels of spin $I^{\prime}$ at excitation energy $U^{\prime}$ in the residual nucleus. All energetically possible final channels $\chi^{\prime \prime}$ have to be taken into account, thus the denominator includes contributions from decays to discrete levels in the residual nuclei (given by the first sum in the denominator, $\Sigma^{\prime}$ ) as well as contributions from decays to regions of high level density in the residual nuclei (given by the second sum in the denominator which involves an energy integral of transmission coefficients and level densities in the residual nuclei). For simplicity, the parity quantum numbers have been suppressed in Equation 1. In realistic applications of 
the Hauser-Feshbach formalism, the level density depends on parity (even though this dependence tends to be weak), and all sums over quantum numbers respect parity conservation.

The above Hauser-Feshbach formula neglects correlations between the incident and outgoing reaction channels. These correlations can be taken into account formally by including width fluctuation corrections $W_{\alpha \chi}$ in the HauserFeshbach formula. The primary effect of the correlations is an enhancement of the elastic scattering cross section. Due to the requirement of flux conservation the inelastic and reaction cross sections are reduced, although this depletion rarely exceeds $10-20 \%$, even at relatively low energies (below approximately $2 \mathrm{MeV}$ ). As the excitation energy of the compound nucleus increases and many reaction channels become available, the effect of the width fluctuations becomes quickly negligible for the non-elastic channels.

In the remainder of this contribution we will neglect these correlations and set $W_{\alpha \chi}=1$. (The elastic channel will be considered separately wherever necessary.) This then allows us to rewrite the Hauser-Feshbach formula as:

$$
\frac{d \sigma_{\alpha \chi}^{H F}\left(E_{a}\right)}{d E_{\chi}}=\sum_{J \Pi} \sigma_{\alpha}^{C N}\left(E_{e x}, J, \pi\right) G_{\chi}^{C N}\left(E_{e x}, J, \pi\right),
$$

where $\sigma^{C N}\left(E_{e x}, J, \pi\right)=\sigma\left(a+A \rightarrow B^{*}\right)$ denotes the cross section for forming the compound nucleus at excitation energy $E_{e x}$ with angular-momentum and parity quantum numbers $J \pi$ and $G_{\chi}^{C N}\left(E_{e x}, J, \pi\right)$ is the branching ratio for the decay of this compound state into the desired exit channel $\chi$.

\subsection{Hauser-Feshbach Formulation of the Surrogate Method}

In the limit of negligible width fluctuation corrections considered here, the formation and decay of the compound nucleus are independent of each other, individually for each angular momentum and parity value. It is this independence that allows one to determine the desired cross section via a combination of theory and experiment in the Surrogate approach. In many cases the formation cross section $\sigma_{\alpha}^{C N}$ can be calculated to a reasonable accuracy by using optical potentials while the theoretical branching ratios $G_{\chi}^{C N}$ for the different channels $\chi$ are often quite uncertain. The objective of the Surrogate method is to determine or constrain these decay probabilities experimentally.

In a Surrogate experiment, the compound nucleus $B^{*}$ is produced via an alternative (Surrogate), direct reaction $d+D \rightarrow b+B^{*}$ and the decay of $B^{*}$ is observed in coincidence with the outgoing particle $b$. The direct-reaction particle is typically stopped in a detector which provides particle identification, as well as information on the kinetic energy and direction of $b$. The desired exit channel $\chi$ can be identified, e.g., by detecting fission fragments from $B^{*}$ or $\gamma$ rays from the desired residual nucleus $C$. The probability for forming $B^{*}$ in the Surrogate reaction (with specific values for the excitation energy $E_{e x}$, angular momentum $J$, and parity $\pi$ ) is $F_{\delta}^{C N}\left(E_{e x}, J, \pi\right)$, where $\delta$ refers to the entrance channel $d+D$. The quantity

$$
P_{\delta \chi}\left(E_{e x}\right)=\sum_{J, \pi} F_{\delta}^{C N}\left(E_{e x}, J, \pi\right) G_{\chi}^{C N}\left(E_{e x}, J, \pi\right),
$$

which gives the probability that the compound nucleus $B^{*}$ was formed with energy $E_{e x}$ and decayed into channel $\chi$, can be obtained experimentally. The direct-reaction probabilities $F_{\delta}^{C N}\left(E_{e x}, J, \pi\right)$ have to be determined theoretically, so that the branching ratios $G_{\chi}^{C N}\left(E_{e x}, J, \pi\right)$ can be constrained from the measurements. In practice, the decay of the compound nucleus is modeled and the $G_{\chi}^{C N}\left(E_{e x}, J, \pi\right)$ are obtained by fitting the calculations to reproduce the measured decay probabilities $P_{\delta \chi}\left(E_{e x}\right)$. Subsequently, the branching ratios obtained in this manner are inserted in Equation (2) to yield the desired reaction cross section. For simplicity, we have omitted the angular dependence of both the desired and the Surrogate reactions in the above discussion. The extension of the Hauser-Feshbach formulae is straight-forward [8].

\subsection{Challenges for the Surrogate Method}

In practice the procedure of determining the branching ratios is a difficult task due to several theoretical and experimental challenges: i) The experimental determination of the decay probability $P_{\delta \chi}\left(E_{e x}\right)=N_{\delta \chi} / N_{\delta}$ requires that both the number of $b-\chi$ coincidences, $N_{\delta \chi}$, and the number of reaction events, $N_{\delta}$ are accurately determined. If target contaminants are present, it becomes very difficult, if not impossible to determine a reliable value for $N_{\delta}$. ii) The theoretical prediction of the direct-reaction probabilities $F_{\delta}^{C N}\left(E_{e x}, J, \pi\right)$ requires a framework for calculating cross 
sections of direct reactions (stripping, pick-up, and inelastic scattering) to continuum states in $B^{*}$. iii) Extracting the branching ratios from measured decay probabilities $P_{\delta \chi}\left(E_{e x}\right)$ requires modeling the decay of the compound nucleus produced in the Surrogate reaction and fitting the relevant parameters to reproduce the experimental results. iv) The possibility that the intermediate nucleus produced in the Surrogate reaction decays before statistical equilibrium is reached $[9,13,14]$ has to be excluded or minimized.

\section{THE WEISSKOPF-EWING LIMIT}

The Hauser-Feshbach theory used in the previous section rigorously conserves total angular momentum $J$ and parity $\pi$. Under certain conditions the branching ratios $G_{\chi}^{C N}\left(E_{e x}, J, \pi\right)$ can be treated as independent of $J$ and $\pi$ and the form of the cross section (for the desired reaction) simplifies to:

$$
\frac{d \sigma_{\alpha \chi}^{W E}\left(E_{a}\right)}{d E_{\chi}}=\sigma_{\alpha}^{C N}\left(E_{e x}\right) \mathscr{G}_{\chi}^{C N}\left(E_{e x}\right)
$$

where

$$
\sigma_{\alpha}^{C N}\left(E_{e x}\right)=\sum_{J \Pi} \sigma_{\alpha}^{C N}\left(E_{e x}, J, \pi\right)=\pi \tau_{\alpha}^{2} \sum_{l=0}^{\infty}(2 l+1) T_{\alpha l}
$$

is the reaction cross section describing the formation of the compound nucleus at energy $E_{e x}$ and $\mathscr{G}_{\chi}^{C N}\left(E_{e x}\right)$ denotes the $J \pi$-independent branching ratio for the exit channel $\chi$. This is the Weisskopf-Ewing limit of the Hauser-Feshbach theory. It is applicable when the following conditions are satisfied $[9,10]$ :

- The energy of the compound nucleus has to be sufficiently high, so that almost all channels into which the nucleus can decay are dominated by integrals over the level density (i.e. the first sum in the denominator of Equation 1, $\Sigma^{\prime}$ has to be negligible).

- Width fluctuations have to be negligible. This will be the case if the previous condition is satisfied.

- The transmission coefficients $T_{\chi^{\prime \prime} l^{\prime \prime} s^{\prime \prime}}^{J}$ associated with the available exit channels have to be independent of the spin of the states reached in these channels. This condition is known to be satisfied since the dependence of transmission coefficients on target spin is generally weak.

- The level densities $\rho_{I^{\prime \prime}}\left(U^{\prime \prime}\right)$ in the available channels have to be independent of parity and their dependence on the spin $I^{\prime \prime}$ of the relevant nuclei has to be of the form $\rho_{I^{\prime \prime}}\left(U^{\prime \prime}\right) \propto\left(2 I^{\prime \prime}+1\right)$. Using tools from statistical mechanics, it can be shown that for sufficiently high excitation energies $U^{\prime \prime}$, level densities are very weakly dependent on parity, so that the first of these conditions can be assumed to be satisfied. The second condition, which is a prerequisite for a rigorous derivation of the Weisskopf-Ewing limit from the full Hauser-Feshbach theory, is satisfied if the spin $I^{\prime \prime}$ is smaller than the spin cutoff parameter $\sigma_{\text {cut }}$ in the relevant level density formula. In some cases, the spin cutoff parameter is not very large (e.g. $\sigma_{c u t} \approx 6-7$ in the actinide region) but it is known empirically that the Weisskopf-Ewing limit is still roughly correct at higher spins.

The cross section in Equation 4 is expressed in differential form, with respect to the energy in the decay channel. For a comparison with a measurement the cross section needs to be integrated over all final-state energies, i.e. the quantity $\mathscr{G}_{\chi}^{C N}$ appearing on the right side has to be integrated over the energy $E_{\chi}$. In the following only integrated quantities will be considered; the energy differential will be removed from the cross section expressions and the $\mathscr{G}_{\chi}^{C N}$ will represent integrated branching ratios.

\subsection{The Surrogate Method in the Weisskopf-Ewing Limit}

The Weiskopf-Ewing limit provides a simple and powerful approximate way of calculating cross sections for twostep reactions proceeding through a compound nucleus. In the context of Surrogate reactions, it greatly simplifies the application of the method: It becomes straightforward to obtain the $J \pi$-independent branching ratios $\mathscr{G}_{\chi}^{C N}\left(E_{e x}\right)$ from measurements, since $P_{\delta \chi}\left(E_{e x}\right)=\mathscr{G}_{\chi}^{C N}\left(E_{e x}\right) \sum_{J \pi} F_{\delta}^{C N}\left(E_{e x}, J, \pi\right)=\mathscr{G}_{\chi}^{C N}\left(E_{e x}\right)$, and to calculate the desired reaction cross 
section. Calculating the direct-reaction probabilities $F_{\delta}^{C N}\left(E_{e x}, J, \pi\right)$ and modeling the decay of the compound nucleus are no longer required.

A Surrogate analysis in the Weisskopf-Ewing approximation was used in the 1970s to extract $(n, f)$ cross sections for various actinides from transfer reactions with $t$ and ${ }^{3} \mathrm{He}$ projectiles on neighboring nuclei, followed by fission [6]. Measured fission probabilities, $P_{f}$, were multiplied by an estimated cross section for the formation of the compound nucleus in the neutron-induced reaction of interest: $\sigma_{(n, f)} \approx \sigma_{n}^{C N} P_{f}$. The resulting $(n, f)$ cross section estimates agreed with direct measurements (where available) to about 10-20\% for incident neutron energies above about $1 \mathrm{MeV}$, but exhibited serious discrepancies below $1 \mathrm{MeV}$, which were attributed to i) large uncertainties in the low-energy optical-model calculations employed, and ii) the neglect of the difference in the angular-momentum populations of the compound nucleus in the Surrogate (direct) and "desired" (neutron-induced) reactions. A more recent analysis of the data used a simple direct-reaction model to account for the angular-momentum difference between the neutron-induced and direct reactions, i.e. it employed the full Surrogate framework, as well as improved optical-model calculations [11]. The results showed significant improvements over the earlier work in the Weisskopf-Ewing approximation.

The assumption that the Weisskopf-Ewing limit is valid implies a significant restriction for possible applications. At low excitation energies in the compound nucleus, the Weisskopf-Ewing description is not even approximately valid. For example, astrophysical $(n, \gamma)$ reactions on s-process branch points cannot be estimated with this method since the relevant neutron energies are very low, $E_{n} \lesssim 100 \mathrm{keV}$, i.e. the compound nucleus is excited to only slightly above the neutron separation threshold. At higher energies the assumption of $J \pi$-independent branching ratios, $G_{\chi}^{C N}\left(E_{e x}, J, \pi\right)$ $\approx \mathscr{G}_{\chi}^{C N}\left(E_{e x}\right)$, breaks down for angular-momentum values larger than the relevant spin-cutoff parameter. This may affect the desired and Surrogate reactions differently, since the angular-momentum transferred is in general larger in the direct reaction than in the compound-nucleus reaction. For example, a Weisskopf-Ewing description might be applicable to an $n$-induced reaction on a target with small spin, while the Surrogate reaction that produces the same compound nucleus might populate states with spins much larger than the spin-cutoff parameter. In such situations a full Surrogate analysis, which takes into account conservation of spin and parity, is required.

Despite the simplifications that can be obtained in the Weisskopf-Ewing description, a number of issues remain to be resolved when applying this approximate version of the Surrogate approach. First, it is not a priori clear whether the Weisskopf-Ewing limit applies to a particular reaction in a given energy regime. This needs to be verified for each case of interest. In addition, even in the Weisskopf-Ewing limit it is necessary to consider the possibility that the intermediate nucleus which is produced in the Surrogate reaction can decay before it reaches equilibrium. Furthermore, Surrogate experiments in the Weisskopf-Ewing limit are still challenging since the requirement that both the number of $b-\chi$ coincidences and the number of reaction events be accurately determined remains.

\section{RATIOS OF SURROGATE MEASUREMENTS}

In a recent publication [5], a new approach was employed with the goal of determining the neutron-induced fission cross section for ${ }^{237} \mathrm{U}$, for neutron energies up to approximately $14 \mathrm{MeV}$. This new approach, which we shall refer to as the "Ratio method", makes use of the Surrogate idea and requires the validity of the Weisskopf-Ewing limit. The primary motivation for using the Ratio method is the fact that it eliminates the need to accurately measure $N_{\delta}$, the total number of reaction events, which has been the source of the largest uncertainty in Surrogate experiments performed recently.

The goal of the Ratio method is to experimentally determine the ratio

$$
R(E)=\frac{\sigma_{\alpha_{1} \chi_{1}}}{\sigma_{\alpha_{2} \chi_{2}}}
$$

of the cross sections of two compound-nucleus reactions, $a_{1}+A_{1} \rightarrow B_{1}^{*} \rightarrow c_{1}+C_{1}$ and $a_{2}+A_{2} \rightarrow B_{2}^{*} \rightarrow c_{2}+C_{2}$, where the two reactions have to be "similar" in a sense that remains to be specified. An independent determination of one of these cross sections then allows one to infer the other by using the ratio $R$. In the Weisskopf-Ewing limit, the ratio $R$ can be written as

$$
R(E)=\frac{\sigma_{\alpha_{1}}^{C N}(E) \mathscr{G}_{\chi_{1}}^{C N}(E)}{\sigma_{\alpha_{2}}^{C N}(E) \mathscr{G}_{\chi_{2}}^{C N}(E)}
$$


with branching ratios $\mathscr{G}_{\chi}^{C N}$ that are independent of the $J \pi$ population of the compound nuclei under consideration. For most cases of interest the compound-nucleus formation cross sections $\sigma_{\alpha_{1}}^{C N}$ and $\sigma_{\alpha_{2}}^{C N}$ can be calculated sufficiently reliably by using an optical model.

To determine $\mathscr{G}_{\chi_{1}}^{C N} / \mathscr{G}_{\chi_{2}}^{C N}$, two experiments are carried out. Both use the same direct-reaction mechanism, $D(d, b) B^{*}$, but different targets, $D_{1}$ and $D_{2}$, to create the relevant compound nuclei, $B_{1}^{*}$ and $B_{2}^{*}$, respectively. For each experiment, the number of coincidence events, $N_{\delta_{1} \chi_{1}}^{(1)}$ and $N_{\delta_{2} \chi_{2}}^{(2)}$, is measured. The ratio of the branching ratios, for decay into the desired channel, for the compound nuclei created in the two reactions is given by:

$$
\frac{\mathscr{G}_{\chi_{1}}^{C N}(E)}{\mathscr{G}_{\chi_{2}}^{C N}(E)}=\frac{N_{\delta_{1} \chi_{1}}^{(1)}(E)}{N_{\delta_{2} \chi_{2}}^{(2)}(E)} \times \frac{N_{\delta_{2}}^{(2)}(E)}{N_{\delta_{1}}^{(1)}(E)} .
$$

In the Ratio approach the experimental conditions are adjusted such that both experiments give the same number of reaction events, $N_{\delta_{1} \chi_{1}}^{(1)} \approx N_{\delta_{2} \chi_{2}}^{(2)}$. This requires that the same setup be used in both experiments. Furthermore, the beam intensities and beam times have to be the same in both cases, and the number of atoms in each target must be equal or the differences have to be accounted for in the data analysis. Under those conditions, the ratio of the branching ratios simply equals the ratio of the coincidence events and the quantity $R$ becomes:

$$
R(E)=\frac{\sigma_{\alpha_{1}}^{C N}(E) N_{\delta_{1} \chi_{1}}^{(1)}(E)}{\sigma_{\alpha_{2}}^{C N}(E) N_{\delta_{2} \chi_{2}}^{(2)}(E)}
$$

The definition of the energy $E$ in the above equations remains to be specified. Typically, the energy-dependence of a compound-nucleus formation cross section, $\sigma_{\alpha}^{C N}=\sigma\left(a+A \rightarrow B^{*}\right)$ is characterized by the kinetic energy of the projectile, $E_{a}$, while a branching ratio is normally given as a function of the excitation energy of the compound nucleus, $\mathscr{G}_{\chi}^{C N}\left(E_{e x}\right)$. In a compound-nucleus reaction, those two values are related via the separation energy $S_{a}$ of the particle $a$ in $B^{*}: E_{e x}=S_{a}+E_{a}$. While either $E_{e x}$ or $E_{a}$ can be used to uniquely specify the energy-dependence of such a reaction, it is important for the Ratio method that the comparison of the relevant reactions, $a_{1}+A_{1} \rightarrow B_{1}^{*} \rightarrow c_{1}+C_{1}$ and $a_{2}+A_{2} \rightarrow B_{2}^{*} \rightarrow c_{2}+C_{2}$, be made at the same projectile energy $E_{a}$. For a given projectile energy, $E_{a_{1}}=E_{a_{2}}$, small differences in the separation energies, $S_{a_{1}}$ and $S_{a_{2}}$, will lead to different excitation energies in the compound nuclei, $B_{1}^{*}$ and $B_{2}^{*}$, respectively. In typical applications of interest the branching ratios are less sensitive to such variation in excitation energy than the formation cross sections are to an energy variation of this magnitude. Thus, in the context of the Ratio approach, we take $E$ to denote the kinetic energy of the projectile.

An illustration of the Ratio method. In Ref. [5], the Ratio method was used to obtain an estimate of the ${ }^{237} \mathrm{U}(\mathrm{n}, \mathrm{f})$ cross section up to approximately $14 \mathrm{MeV}$. To this end, the ratio

$$
R=\frac{\sigma\left({ }^{237} U(n, f)\right)}{\sigma\left({ }^{235} U(n, f)\right)} \approx \frac{\sigma_{n+{ }^{237} U}^{C N} \mathscr{G}_{238}^{C N} U \rightarrow f}{\sigma_{n+{ }^{235} U}^{C N} \mathscr{G}_{236}^{C N} U \rightarrow f}
$$

was considered, where the cross section $\sigma\left({ }^{235} U(n, f)\right)$ for neutron-induced fission of ${ }^{235} \mathrm{U}$ is known. The formation cross sections for the compound nuclei ${ }^{238} \mathrm{U}$ and ${ }^{236} \mathrm{U}$ were assumed to be very similar, $\sigma_{n+}^{C N}{ }^{237} U \sigma_{n+{ }^{235} U}$. To obtain information on the branching ratios $\mathscr{G}_{238}^{C N}$ and $\mathscr{G}_{236}^{C N}$, inelastic deuteron scattering experiments on ${ }^{238} \mathrm{U}$ and ${ }^{236} \mathrm{U}$ were carried out. Fission fragments from ${ }^{238} U\left(d, d^{\prime} f\right)$ and ${ }^{236} U\left(d, d^{\prime} f\right)$ were detected in coincidence with the outgoing deuterons and

$$
\frac{\mathscr{G}_{238}^{C N} \rightarrow f}{\mathscr{G}_{236}^{C N} U \rightarrow f} \approx \frac{N_{238} U\left(d, d^{\prime} f\right)}{N_{236} U\left(d, d^{\prime} f\right)}
$$

was determined. Corrections were applied to account for differences in target thickness and beam intensity. The resulting cross section ratio was found to be in agreement with a theoretical estimate by Younes et al. [12]. 


\subsection{Limitations of the Ratio Approach}

The experiments required for a Ratio analysis are simpler than those that need to be carried out if a full Surrogate analysis (or a Surrogate analysis in the Weisskopf-Ewing limit) is planned. The primary advantage of considering relative branching ratios and relative cross sections lies in the fact that the number of direct-reaction events, $N_{\delta}$, does not need to be determined for a Ratio analysis. Furthermore, unlike in the full Surrogate treatment, it is not necessary to calculate the direct-reaction probabilities, $F_{\delta}^{C N}(E, J, \pi)$, or to model the decay of the compound nucleus.

The Ratio method is based on the assumption that the Weisskopf-Ewing approximation is valid. It is therefore subject to the same restrictions that apply to the use of a Surrogate analysis in the Weisskopf-Ewing approximation, although small deviations from this assumption might affect the Ratio analysis to a lesser extent.

The Ratio method is also limited by the requirement that for obtaining an absolute result for an unknown cross section $\sigma\left(a_{1}+A_{1} \rightarrow B_{1}^{*} \rightarrow c_{1}+C_{1}\right)$ a reliable independent cross-section measurement for a similar reaction, $a_{2}+A_{2} \rightarrow$ $B_{2}^{*} \rightarrow c_{2}+C_{2}$, at the same equivalent projectile energies, must be available. Furthermore, it is required that a directreaction mechanism, $D(d, b) B^{*}$, and target-projectile combinations can be identified that make it possible to produce the compound nuclei, $B_{1}^{*}$ and $B_{2}^{*}$, respectively.

One can expect reliable cross section estimates from the Ratio approach only when the two reactions that are analyzed, $D_{1}(d, b) B_{1}^{*}$ and $D_{2}(d, b) B_{2}^{*}$, are sufficiently similar. When small systematic errors or small violations of the prerequisite assumptions, such as the validity of the Weisskopf-Ewing approximation or the absence of nonequilibrium decays, affect both reactions in the same manner, it is likely that the effects cancel in part in the Ratio analysis. Uncorrelated errors and deviations, on the other hand, will increase the overall uncertainty in the final result. Similarity in the present context implies that i) the same projectile initiates the compound-nucleus reactions that are compared, i.e. $a_{1}=a_{2}$, and the same kind of decay (gamma emission, charged-particle emission, or fission) is considered in both cases; ii) the decays of the compound nuclei $B_{1}^{*}$ and $B_{2}^{*}$ have similar properties (number and kind of open channels, separation energies for the various channels, level densities in the residual nuclei, etc.); iii) the direct (Surrogate) reactions which produce the compound nuclei employ the same mechanism, $D(d, b) B^{*}$, and projectileejectile combination, $d-b$, in both cases.

\section{CONCLUDING REMARKS}

Indirect methods play an important role for obtaining many reaction cross sections of interest. A method which aims at extracting cross sections for reactions proceeding through a compound nucleus has been presented. While the Surrogate method is very general and can in principle be employed to determine cross sections of all types of compound-nucleus reactions on a large variety of nuclei, significant challenges remain to be addressed to establish the validity of this approach. For applications to $(\mathrm{n}, \mathrm{f})$ cross sections on actinide nuclei, Younes and Britt have studied some of these issues [11] and for applications to ( $\mathrm{n}, \gamma)$ cross sections on lighter nuclei, work is currently underway [7]. In the interim, it is useful to investigate whether certain simplifications or approximations to the method can be utilized to determine the relevant cross sections. Two possible approximations to the Surrogate method have been considered here, the Weisskopf-Ewing limit of the Surrogate method and the Ratio approach. Both provide simple and potentially powerful ways of estimating cross sections that cannot be measured directly. Both are much simpler than a full Surrogate treatment and both are much more limited in their applicability.

\section{ACKNOWLEDGMENTS}

The authors gratefully acknowledge receiving a copy of Ref. [5] prior to publication. This work was performed under the auspices of the U.S. Department of Energy by the University of California, Lawrence Livermore National Laboratory (LLNL) under contract No. W-7405-Eng-48. Partial funding was provided by the Laboratory Directed Research and Development (LDRD) Program at LLNL under project 04-ERD-057.

\section{REFERENCES}

1. G. Baur, K. Hencken, and D. Trautmann, Prog. Part. Nucl. Phys. 51487 (2003);

G. Baur and H. Rebel, Annu. Rev. Nucl. Part. Sci. 46321 (1996); 
2. N.K. Timofeyuk, R.C. Johnson, and A.M. Mukhamedzhanov Phys. Rev. Lett. 91232501 (2003);

L. Trache et al., Phys. Rev. C 69032802 (R) (2004);

L. Trache et al., Phys. Rev. Lett. 87271102 (2001);

A. Azhari et al., Phys. Rev. Lett. 823960 (1999);

A.M. Mukhamedzhanov and R.E. Tribble, Phys. Rev. C 593418 (1999);

H. M. Xu et al., Phys. Rev. Lett. 732027 (1994).

3. S. Typel and G. Baur, Ann. Phys. 305228 (2003);

G. Baur and S. Typel, preprint nucl-th/0401054.

4. L. Bernstein, private communication.

5. C. Plettner et al., Phys. Rev. C in press (2005).

6. J.D. Cramer and H.C. Britt, Nucl. Sci. and Eng. 41177 (1970);

J.D. Cramer and H.C. Britt, Phys. Rev. C 22350 (1970);

B.B. Back et al. , Phys. Rev. C 91924 (1974);

B.B. Back et al. , Phys. Rev. C 101948 (1974);

H.C. Britt and J.B. Wilhelmy, Nucl. Sci. and Eng. 72222 (1979).

7. J. Escher et al., Nucl. Phys. A, in press;

C. Forssén et al., Nucl. Phys. A, in press

8. P. Fröbrich and R. Lipperheide, Theory of Nuclear Reactions, Oxford University Press, Oxford, U.K., 1996.

9. E. Gadioli and P. E. Hodgson, Pre-Equilibrium Nuclear Reactions (Clarendon Press, Oxford, 1992).

10. F. S. Dietrich, Simple Derivation of the Hauser-Feshbach and Weisskopf-Ewing Formulae, with Application to Surrogate Reactions, Technical Report No. UCRL-TR-201718, Lawrence Livermore National Laboratory, Livermore, CA, 2004 (unpublished).

11. W. Younes W and H.C. Britt, Phys. Rev. C 67024610 (2003);

W. Younes W and H.C. Britt, Phys. Rev. C 68034610 (2003).

12. W. Younes, H. C. Britt, J. A. Becker, and J. B. Wilhelmy, Initial estimate for the ${ }^{237} U(n, f)$ cross section for $0.1 \leq E_{n}$, Technical Report No. UCRL-ID-154194, Lawrence Livermore National Laboratory, Livermore, CA, 2003 (unpublished).

13. A. K. Kerman and K. W. McVoy, Ann. Phys. (N.Y.) 122197 (1979).

14. W. Parker et al., Phys. Rev. C 52252 (1995). 